\title{
A validated composite organ and hematologic response model for early assessment of treatment outcomes in light chain amyloidosis
}

\author{
Surbhi Sidana $\mathbb{1}^{1,2}$, Paolo Milani ${ }^{3,4}$, Moritz Binder (10), Marco Basset ${ }^{3,4}$, Nidhi Tandon ${ }^{1}$, Andrea Foli ${ }^{3,4}$, Angela Dispenzieri ${ }^{1}$, \\ Morie A. Gertz', Suzanne R. Hayman', Francis K. Buadi', Martha Q. Lacy ${ }^{1}$, Prashant Kapoor ${ }^{1}$, Nelson Leung (1)', \\ S. Vincent Rajkumar (1)', Giampaolo Merlini ${ }^{2,3}$, Giovanni Palladini ${ }^{3,4}$ and Shaji K. Kumar (1)
}

\begin{abstract}
Newly diagnosed AL amyloidosis patients were evaluated to develop a model for early assessment of treatment benefit at 6 months, integrating both hematologic (HR) and organ response (OR) assessment (testing cohort, Mayo: $n=473$; validation cohort, Pavia: $n=575)$. Multiple OR were assessed as follows: All OR (AOR): response in all organs, mixed OR (MOR): response in some organs, no OR (NOR)]. AOR rates at 6 months improved with deepening HR; complete response (CR; 38\%, 35\%), very good partial response (VGPR; 30\%, 26\%), and partial response (PR; 16\%, 21\%), respectively. A composite HR/OR (CHOR) model was developed using incremental scoring based on hazard ratios with scores of 0-3 for HR (0-CR, 1-VGPR, 2-PR, 3-no response) and 0-2 for OR (0-AOR, 1-MOR, 2-NOR). Patients could be divided into two distinct CHOR groups (scores $0-3$ and 4-5), with median OS in group 1 and group 2: Not reached vs. 34 months, $p<0.001$ [Mayo] and 87 vs. 23 months, $p<0.001$ [Pavia]. In conclusion, we developed a model that can assess multiple organs concurrently, and integrate both HR and OR assessments to determine early clinical benefit with treatment, which may be used as a surrogate end-point in trials and to compare outcomes with different therapies.
\end{abstract}

\section{Introduction}

Deposition of misfolded light chains secreted by the plasma cell clone leads to organ dysfunction in patients with light chain (AL) amyloidosis ${ }^{1-3}$. The most commonly affected organs include the heart, kidney, and liver; and many patients have more than one organ involvement ${ }^{3-5}$. Prognosis depends both on the severity of organ involvement, especially the heart, and the underlying plasma cell burden ${ }^{6-8}$. Treatment is targeted toward the plasma cell clone $e^{2,9-13}$. In most patients, the organ dysfunction is the main driver of morbidity and mortality and the plasma

\footnotetext{
Correspondence: Giovanni Palladini (giovanni.palladini@unipv.it) or Shaji K. Kumar (kumar.shaji@mayo.edu)

${ }^{1}$ Division of Hematology, Department of Internal Medicine, Mayo Clinic, Rochester, MN, USA

${ }^{2}$ Department of Medicine, Stanford University, Stanford, CA, USA

Full list of author information is available at the end of the article These authors contributed equally: Surbhi Sidana, Paolo Milani,

Giovanni Palladini
}

cell burden is usually low $w^{6,8,14,15}$. Given this, it would be ideal to assess treatment efficacy by its impact on organ improvement. However, time to organ response (OR) can be varied and is usually delayed ${ }^{5}$. Therefore, treatment efficacy, especially early-on is typically determined by hematologic response $(\mathrm{HR})^{16}$.

Deep HR increases the likelihood of OR and long-term survival, but this is not always the case and there is interpatient variability in the relationship between depths of $\mathrm{HR}$ and $\mathrm{OR}^{5,17}$. No model currently exists to integrate the two assessments for clinical use. This makes early assessment of treatment benefit difficult in this disease, preventing relatively rapid evaluation of clinical trial results and precludes design of clinical trials for timely intervention in patients with likely poor outcome with ongoing therapies. Early identification of patients who are not likely to benefit from a given therapy is increasingly important as these patients have an inferior survival and 
more treatment options are becoming available for these patients ${ }^{3}$. A composite model that takes into account both $\mathrm{HR}$ and $\mathrm{OR}$ at a given time point may allow for early assessment and thus become a useful surrogate endpoint for clinical trials. It may also identify patients who have not achieved a deep HR, but can safely continue first line therapy if they have achieved an OR. Such a surrogate model may also be helpful in light of new, emerging therapies that target the amyloid fibril and can potentially lead to earlier $\mathrm{OR}^{18,19}$.

In this study, we have developed and validated a composite model to integrate $\mathrm{OR}$ and $\mathrm{HR}$ in $\mathrm{AL}$ amyloidosis to define a surrogate end point for use in treatment trials in $\mathrm{AL}$ amyloidosis.

\section{Patients and methods Study population}

Patients with biopsy proven newly diagnosed AL amyloidosis with involvement of heart, liver, or kidney who received treatment were included. Amyloid deposits were confirmed as AL type by electron microscopy immunohistochemistry ${ }^{20}$ or mass spectrometry ${ }^{21}$. We identified 875 patients diagnosed from $1 / 1 / 2006$ to $12 / 31 / 2015$ from the Mayo Clinic dataset, of which 473 patients had HR and OR data available at 6 months (test cohort; Supplementary Fig. 1 consort flow diagram). The validation cohort included 575 patients from the Pavia Amyloidosis Research and Treatment Center dataset. Both datasets are maintained prospectively with approval by the respective Institutional Review Committees.

\section{Response assessment}

In evaluable patients, HR was assessed using validated criteria ${ }^{16}$. Patients who had difference in involved and uninvolved free light chains (dFLC) $<5 \mathrm{mg} / \mathrm{dL}$ were assessed for response by criteria of complete response (CR) only, based on recent data ${ }^{22-24}$. Organ involvement and response were assessed by using existing criteria as described in supplementary data ${ }^{16,25-27}$. OR was classified as all organ response (AOR): response in all of the involved and evaluable organs (heart, kidney, liver); mixed organ response (MOR): response in at least one of the organs and no organ response (NOR). Patients were assessed for response at the 6 months ( \pm 2 months) and 12 months $( \pm 2$ months) time-point in the Mayo cohort and at 6 months time-point ( \pm 2 months) for the Pavia group.

\section{Combined hematologic and OR (CHOR) model}

A model for CHOR was developed using the Mayo Clinic test cohort (Fig. 1). Patients were assigned scores of 0-3 for HR as follows: 0-CR, 1-very good partial response (VGPR), 2-partial response (PR), 3-no response (NR) or progression. Patients who had dFLC $<5 \mathrm{mg} / \mathrm{dL}$ were assigned a score of 0 for $\mathrm{CR}$ and 1 for

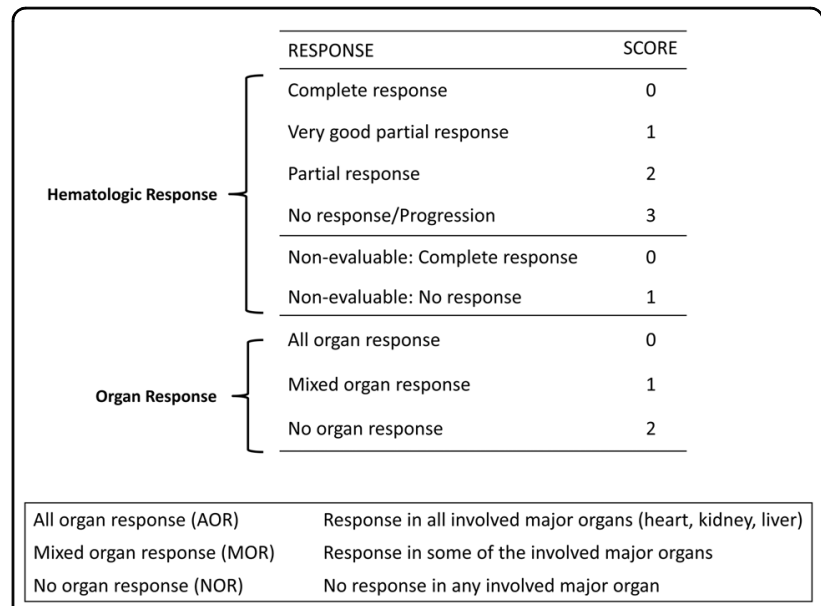

Fig. 1 Scoring system for composite hematologic and organ response (CHOR) model.

other response as OS for the latter group was most similar to achieving VGPR. OR was scored as follows: $0-\mathrm{AOR}, 1$ -MOR, and 2-NOR. Hazard ratios (HR) for OS were calculated for scores 1-5 relative to a score 0 (complete OR and HR) to construct groups based on similar hazard ratios. Patients were then divided into two groups: CHOR group 1 (scores $0-3$ ) and CHOR group 2 (scores 4-5).

\section{Analysis}

Statistical analysis was carried out using the JMP (version 12, SAS Institute Inc., Cary, NC) and Stata (version 13.1, StataCorp, College Station, TX) software for the Mayo Clinic cohort and using MedCalc Statistical Software version 18.1 (MedCalc Software bvba, Ostend, Belgium; http://www.medcalc.org) for the Pavia cohort. ChiSquare and Fischer Exact tests were used to carry out univariate analysis for categorical variables and Wilcoxon Rank Sum/Kruskal-Wallis for continuous variables. Survival analysis was carried out using the Kaplan-Meier method and the log-rank test was used to compare survival curves. Cox proportional hazards model was used to evaluate hazard ratios for survival. 95\% confidence intervals (CI) are reported. OS was defined as the time from start of treatment to death. Cox regression was used to compare the predictive power of HR, OR, and the composite CHOR model ${ }^{28}$. Goodness of fit of nested models was evaluated using likelihood ratio tests. Predictive power of the individual and composite models were compared using Harrell's $\mathrm{C}^{29}$. All hypothesis tests were two-sided, $p$ values below 0.05 were considered statistically significant.

\section{Results \\ Baseline characteristics}

The test cohort consisted of 473 patients from Mayo Clinic cohort, who were alive at the 6-month time point 
and had HR and OR data available. The validation cohort consisted of 575 patients from Pavia with response data available at the 6-month time-point. Baseline characteristics of patients in the two cohorts are summarized in Table 1 . The median age at diagnosis was $63-64$ years in both series, and males comprised $65 \%$ and $58 \%$ of the population in the Mayo and Pavia cohorts, respectively. Amyloidogenic light chain was lambda in $78 \%$ of patients in both cohorts. Median dFLC and bone marrow plasma cells at diagnosis were: $19 \mathrm{mg} / \mathrm{dL}$ and 10\% (Mayo) and $19 \mathrm{mg} / \mathrm{dL}$ and 11\% (Pavia), respectively. Proportion of patients with $\mathrm{dFLC}<5 \mathrm{mg} / \mathrm{dL}$ (or $50 \mathrm{mg} / \mathrm{L}$ ) at diagnosis was $13 \%$ in the Mayo cohort and $7 \%$ in the Pavia cohort. Presence of $t(11,14)$ on interphase fluorescence in-situ hybridization (iFISH) was noted in $53 \%$ of patients and presence of trisomy/tetrasomy in $21 \%$ of patients in the

Table 1 Baseline characteristics and organ involvement.

\begin{tabular}{|c|c|c|}
\hline & $\begin{array}{l}\text { Mayo cohort } \\
N=473 N(\%) \text { or } \\
\text { Median (IQR) }\end{array}$ & $\begin{array}{l}\text { Pavia cohort, } \\
N=575 N(\%) \text { or } \\
\text { Median (IQR) }\end{array}$ \\
\hline Median age, years & $63(56-69)$ & $64(56-70)$ \\
\hline Sex, males & $309(65)$ & $335(58)$ \\
\hline Involved light chain, lambda & $354 / 467(78)$ & $450 / 575(78)$ \\
\hline Median M-protein (g/dL) & $0(0-0.6)$ & $0.8(0-1.5)$ \\
\hline Median dFLC (mg/dL) & $19(8-55)$ & $19(9-52)$ \\
\hline $\mathrm{dFLC}<5 \mathrm{mg} / \mathrm{dL}$ & $63(13)$ & $39(7)$ \\
\hline Bone marrow plasma cells (\%) & $10(5-12)$ & $11(7-20)$ \\
\hline NT-Pro BNP (pg/mL) & $1625(327-4296)$ & $2215(704-5578)$ \\
\hline Troponin- $\mathrm{T}(\mathrm{ng} / \mathrm{mL})$ & $0.02(0.01-0.05)$ & Not available \\
\hline Troponin-I (ng/mL) & Not available & $0.05(0.02-0.13)$ \\
\hline Median $24 \mathrm{~h}$ urine protein (mg) & $2629(375-7008)$ & $2561(392-6590)$ \\
\hline $\begin{array}{l}\text { Median GFR (ml/min/ } \\
\left.1.73 \mathrm{~m}^{2} \mathrm{BSA}\right)\end{array}$ & $65(48-82)$ & $69(49-87)$ \\
\hline $\begin{array}{l}\text { Median alkaline } \\
\text { phosphatase (U/L) }\end{array}$ & $86(69-118)$ & $149(88-217)$ \\
\hline Mayo 2012 stage 1/2/3/4 & $\begin{array}{l}125 / 123 / 115 / 93(27 / \\
27 / 25 / 20)(N=456)\end{array}$ & $\begin{array}{l}109 / 159 / 151 / 111(20 / \\
30 / 28 / 22)(N=530)\end{array}$ \\
\hline Mayo 2004 stage $1 / 2 / 3 a / 3 b$ & $\begin{array}{l}107 / 205 / 108 / 37(23 / \\
45 / 24 / 8)(N=457)\end{array}$ & $\begin{array}{l}78 / 276 / 107 / 69(15 / 52 / \\
20 / 13)(N=530)\end{array}$ \\
\hline Renal stage $1 / 2 / 3(\%)$ & $\begin{array}{l}229 / 183 / 53(49 / 39 / 11) \\
N=465\end{array}$ & $\begin{array}{l}285 / 213 / 68(50 / 37 / 13) \\
N=566\end{array}$ \\
\hline $\mathrm{iFISH}-\mathrm{t}(11 ; 14)$ & $172 / 325(53)$ & Not available \\
\hline iFISH—trisomy/tetrasomy & $67 / 326(21)$ & Not available \\
\hline \multicolumn{3}{|l|}{ Organ involvement } \\
\hline Heart & $332(70)$ & $454(79)$ \\
\hline Kidney & $330(70)$ & $399(69)$ \\
\hline Liver & $66(14)$ & $64(11)$ \\
\hline Gastrointestinal system & $101(21)$ & $13(2)$ \\
\hline Autonomic nervous system & $47(10)$ & $61(10)$ \\
\hline \multicolumn{3}{|l|}{ Major organ involved ${ }^{\mathrm{a}}$} \\
\hline One major organ involved & $255(54)$ & $269(47)$ \\
\hline Two major organs involved & $181(37)$ & $268(46)$ \\
\hline Three major organs involved & $37(8)$ & $38(7)$ \\
\hline $\begin{array}{l}\text { More than one major organ } \\
\text { involved }\end{array}$ & $218(46)$ & $306(53)$ \\
\hline
\end{tabular}

$B S A$ body surface area, $d F L C$ difference in involved and uninvolved free light chains, GFR Glomerular filtration rate, IQR interquartile, iFISH interphase fluorescence in situ hybridization, M-protein monoclonal protein.

${ }^{a}$ Major organs: heart, kidney, and liver.
Mayo cohort. iFISH data of the Pavia cohort were not available. The most common organs involved were the heart (70\% and 79\%) and kidney (70\% and 69\%). Liver was involved in $14 \%$ and $11 \%$ of patients, respectively. Number of major organs (heart, liver, and kidney) involved in patients from the Mayo cohort were as follows, one: $54 \%$, two: $37 \%$, and three: $8 \%$. In the Pavia cohort, distribution of organ involvement was as follows: one: $47 \%$, two: $46 \%$, and three: $7 \%$.

\section{Treatment and Hematologic Response}

First-line treatment received by the 473 patients in the Mayo cohort was as follows: autologous stem cell transplant (ASCT) with or without induction therapy, $41 \%$ $(n=194)$; bortezomib-based chemotherapy, 21\% $(n=$ $100)$; alkylator-based therapy, $35 \%(n=165)$, immunomodulatory drugs $3 \%(n=13)$, and steroids/other in one patient. HR rates at 6 months in the 410 evaluable patients were as follows: CR was seen in 25\% (101/410), VGPR in 35\% (144/410), PR in 24\% (99/410), while 16\% (66/410) of patients had NR/disease progression. There were 63 patients who were not evaluable given baseline $\mathrm{dFLC}<5 \mathrm{mg} / \mathrm{dL}$. Of these, 29\% (18/63) achieved a CR. In the Pavia cohort, treatment received was as follows: bortezomib-based chemotherapy, $44 \%(n=255)$, melphalan and dexamethasone, $40 \%(n=234)$, immunomodulatory drugs $9 \%(n=52)$, ASCT, $1 \%(n=9)$, and steroids/other $4 \%(n=25)$. HR rates at 6 months were: CR: $14 \%$ (74/536), VGPR: 33\% (176/536), PR: 19\% (105/ 536 ), while $34 \%$ of patients had NR/disease progression (181/536). Remaining 39 patients had $\mathrm{dFLC}<5 \mathrm{mg} / \mathrm{dL}$ and all achieved a CR.

\section{Organ Response Rates}

Individual OR For individual OR assessment, patients on dialysis at diagnosis were excluded from renal response assessment (Mayo: 4\%; $n=14$; Pavia: $1 \%, n=9$ ). In the Mayo cohort, cardiac response was seen in 25\% (73/293), renal response in 34\% (103/300), and liver response in $25 \%(16 / 64)$ of patients evaluated at the 6 months timepoint, respectively. Rates of OR at 6 months in the Pavia cohort were: cardiac: $27 \%$ (116/424), renal: 36\% (135/375), and liver: $17 \%$ (9/52), respectively.

Combined OR Combined OR in the Mayo Clinic cohort at 6 months was as follows: AOR 26\% (125/473), MOR: 14\% $(n=66 / 473)$, NOR: $60 \%(n=282 / 473)$. OR rates improved at 12 months; AOR: $45 \%(n=194 / 435)$, MOR: $12 \%(n=54 / 435)$ and NOR: $43 \%(n=187 / 435)$. Combined OR rates at 6 months in the Pavia cohort were as follows: AOR 21\% $(n=120 / 575)$, MOR: $18 \%(n=105 /$ 575), NOR: 61\% $(n=350 / 575)$. Combined OR rates increased with deeper HR. In the Mayo cohort, OR rates at 6 months for patients achieving hematologic $C R$ 
(including CR in $\mathrm{dFLC}<5 \mathrm{mg} / \mathrm{dL}$ ) were: AOR $38 \%$ (45/ 119), MOR: $13 \%$ (15/119), and NOR 50\% (59/119). OR in the hematologic VGPR group (including $\mathrm{dFLC}<5 \mathrm{mg} / \mathrm{dL}$ without CR) were AOR: 30\% (57/189), MOR: 16\% (31/ 189), and NOR: $53 \%$ (101/189) and in the hematologic PR group were AOR: 16\% (16/99), MOR: 18\% (18/99), and NOR 66\% (65/99). In the Pavia cohort, OR rates for patients achieving hematologic CR were: AOR 35\% (40/ 113), MOR: $23 \%$ (26/113), and NOR 41\% (47/113). OR in the hematologic VGPR group were AOR: 26\% (46/176), MOR: $22 \%$ (39/176), and NOR: $51 \%(91 / 176)$ and in the hematologic PR group were AOR: 21\% (22/105), MOR: $20 \%(21 / 105)$, and NOR 59\% (62/105).

In the Mayo Clinic group, there were nine patients who either had no HR or hematologic progression at 6 months, who had concurrent OR. There were three patients with both kidney and renal involvement; two of them had only renal response and one had both heart and renal response. The remaining six patients had renal involvement alone and had a renal response. In the Pavia cohort at 6 months there were seven patients who obtained a cardiac response and 24 patients who had a renal response, while no HR was achieved. However, in all cases a reduction of dFLC between $40 \%$ and $50 \%$ was achieved.

\section{Survival}

Figure 2 and Table 2 describe the survival outcomes in the Mayo cohort based on the combined OR parameter for the 6-month response (Fig. 2a, b). Survival based on combined OR was analyzed for all patients (Fig. 2a) and subsets of patients with more than one organ involved (Fig. 2b). Patients who achieved AOR at 6 months had the best outcomes with median OS in AOR vs. MOR vs. NOR groups being:, not reached vs. 81 vs. 85 months, $p<0.001$. In patients with more than one organ involved, where patients could have mixed or discordant ORs, median OS in the three groups (AOR vs. MOR vs. NOR) was not reached. vs. 81 vs. 52 months. These parameters were evaluated in the Pavia cohort based on 6-month OR and were predictive of OS (Table 2, Fig. 3a, b). Patients with AOR had the best survival, followed by MOR and NOR (Fig. 3a). Subset analyses in patients with more than one organ involved showed similar results (Fig. 3b and Supplementary Fig. 2). While the absolute survival outcomes were different in the Mayo Clinic and Pavia cohort, the survival trend and magnitude of difference was similar across the two groups. In the subset of patients with heart involvement, OS based on AOR vs. MOR vs. NOR was as follows in the Mayo Cohort: not reached vs. 81 months vs. 63 months, $p<0.001$ and similar in the Pavia cohort (Table 2 and Supplementary Fig. 3).

In patients with involvement of both heart and kidney and who achieved cardiac response by 6 months, status of renal response did not impact survival further as shown in Supplementary data. Amongst patients with renal involvement, achievement of renal response by 6 months was associated with significantly better dialysis-free survival, with $88 \%$ vs. $65 \%$ of patients remaining dialysis free at 5 years, $p<0.001$ (Mayo cohort)

\section{CHOR model}

A composite score (CHOR) was developed based on HR and OR as described in the "Methods" section (Fig. 1). Patients with a score of zero were those who achieved a hematologic CR as well as well as response in all organs. Patients were divided into two groups based on the HR for survival (Mayo cohort). The groups were as follows: group 1: scores of $0-3(N=349)$, group 2: scores of $4-5(N=$ 124). As illustrated in Fig. 4a, b, patients in CHOR group 1 had significantly better survival outcome compared to group 2 (median OS: not reached vs. 34 months, $p<$ $0.001)$ with HR of 3.4 (2.5-4.6), $p<0.001$. This model was then validated in the Pavia cohort and median OS for patients in CHOR group 1 vs. 2 was 87 vs. 23 months, $p<$ 0.001 with HR of $2.8(2.2-3.5), p<0.001$. This model was

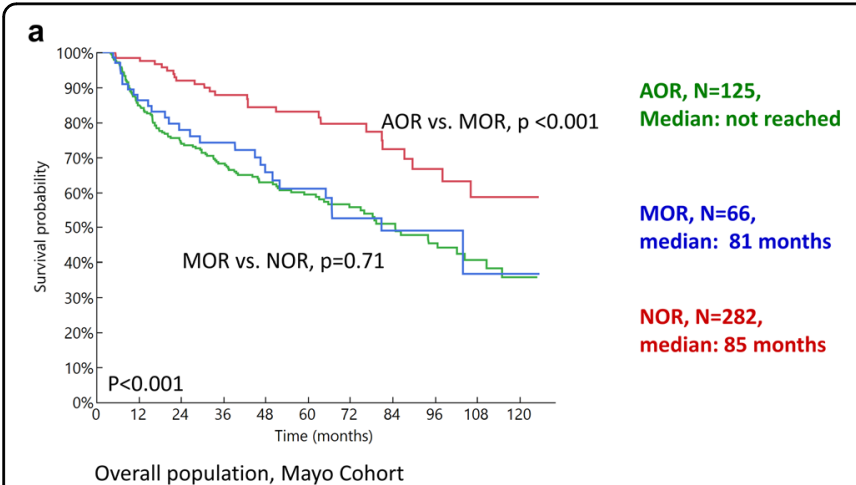

$$
\text { b }
$$

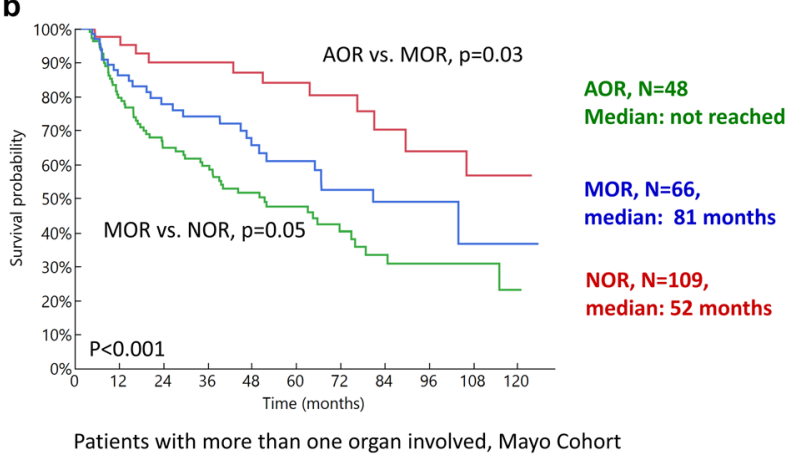

Fig. 2 Overall survival in the Mayo Clinic cohort based on achieving all organ response (AOR), mixed organ response (MOR) and no organ response (NOR) at 6 months from start of first-line therapy. a All patients, b patients with more than one organ involved. 
Table 2 Overall survival outcomes based on combined organ response and composite hematologic and organ response (CHOR) model.

\begin{tabular}{|c|c|c|c|c|c|c|c|c|}
\hline & \multicolumn{4}{|c|}{ Mayo Clinic cohort } & \multicolumn{4}{|c|}{ Pavia cohort } \\
\hline & $N$ & $\begin{array}{l}\text { Median OS } \\
(95 \% \mathrm{Cl})\end{array}$ & $p$-value & $\begin{array}{l}\text { Hazard ratio }(95 \% \mathrm{Cl}) \text {, } \\
p \text {-value }\end{array}$ & $N$ & $\begin{array}{l}\text { Median OS } \\
(95 \% \mathrm{CI})\end{array}$ & $p$-value & $\begin{array}{l}\text { Hazard ratio }(95 \% \mathrm{Cl}) \text {, } \\
p \text {-value }\end{array}$ \\
\hline \multicolumn{9}{|c|}{ Combined organ response } \\
\hline Overall population & 473 & & & & 575 & & & \\
\hline $\mathrm{AOR}$ & 125 & NR (98-NR) & & Reference & 120 & $85(79-100)$ & & Reference \\
\hline MOR & 66 & 81 (50-NR) & $<0.001^{\mathrm{a}}$ & $2.2(1.3-3.8), p=0.003$ & 105 & $62(50-98)$ & $0.028^{\mathrm{a}}$ & $1.6(1.0-2.3), p=0.029$ \\
\hline NOR & 282 & 85 months (66-105) & $0.71^{\mathrm{b}}$ & $2.4(1.6-3.7), p<0.001$ & 350 & $42(33-56)$ & $0.062^{b}$ & $2.1(1.5-2.0), p<0.001$ \\
\hline$>1$ organ involved & 235 & & & & 258 & & & \\
\hline $\mathrm{AOR}$ & 48 & NR (81-NR) & & Reference & 28 & $82(77-100)$ & & \\
\hline MOR & 66 & 81 months (50-NR) & $0.03^{\mathrm{a}}$ & $2.1(1.1-4.0), p=0.02$ & 105 & $62(50-98)$ & $0.130^{\mathrm{a}}$ & $1.6(0.8-3.1), p=0.13$ \\
\hline NOR & 109 & 52 months (35-75) & $0.05^{\mathrm{b}}$ & $2.9(1.7-5.0), p<0.001$ & 125 & $34(23-47)$ & $0.015^{b}$ & $2.6(1.4-5.0), p=0.002$ \\
\hline Heart involvement & 332 & & & & 424 & & & \\
\hline AOR & 68 & NR (81-NR) & & Reference & 72 & $84(73-100)$ & & Reference \\
\hline MOR & 63 & 81 months (50-NR) & $0.05^{\mathrm{a}}$ & $1.8(1.0-3.3), p=0.06$ & 102 & $62(50-98)$ & $0.05^{\mathrm{a}}$ & $1.4(0.9-2-2), p=0.11$ \\
\hline NOR & 201 & 63 months (40-78) & $0.13^{\mathrm{b}}$ & $2.5(1.5-4.2), p<0.001$ & 250 & $25(20-38)$ & $0.001^{b}$ & $2.4(1.6-3.5), p<0.001$ \\
\hline CHOR model & 473 & & & & 575 & & & \\
\hline Group-1 & 349 & NR (103-NR) & & Reference & 344 & 87 (79-98) & & Reference \\
\hline Group-2 & 124 & 34 months (21-46) & $<0.001$ & $3.4(2.5-4.6), p<0.001$ & 231 & $23(18-30)$ & $<0.001$ & $2.8(2.2-3.5), p<0.001$ \\
\hline
\end{tabular}

$A O R$ all organ response, MOR mixed organ response, NOR no organ response, CHOR composite hematologic and organ response, NR not reached, OS overall survival. aLog-rank $p$-value for AOR vs. MOR.

bog-rank $p$-value for MOR vs. NOR.
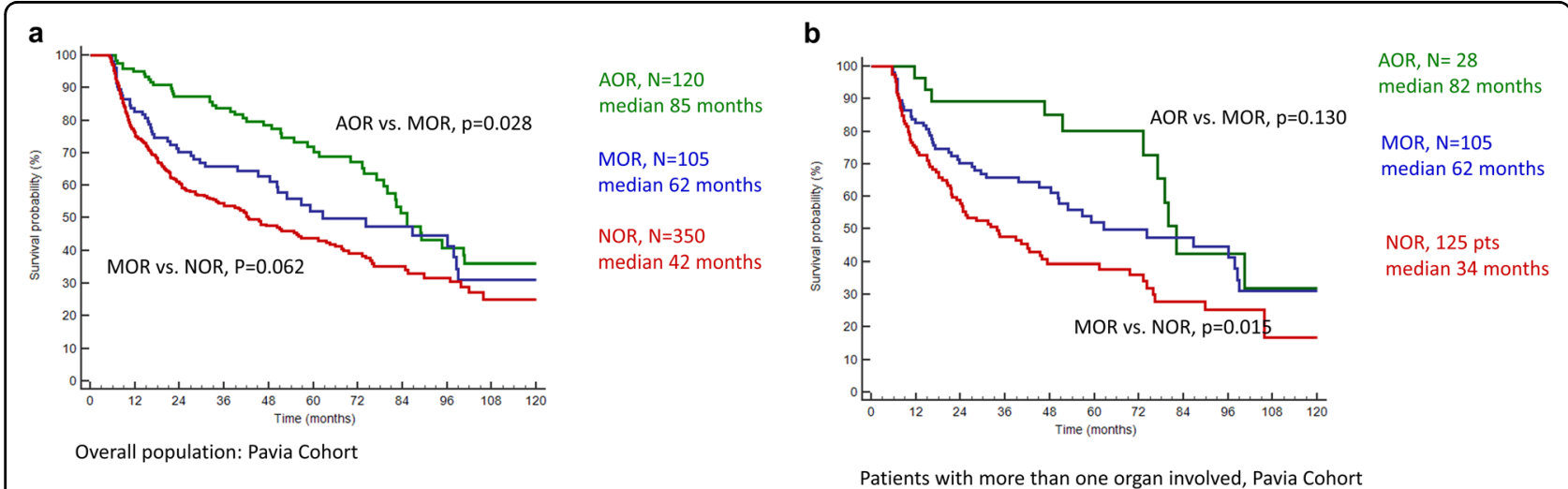

Fig. 3 Overall survival in the Pavia cohort based on achieving all organ response (AOR), mixed organ response (MOR) and no organ response (NOR) at 6 months from start of first-line therapy. a All patients, $\mathbf{b}$ patients with more than one organ involved.

also tested in sub-groups of patients with Mayo 2012 stages I-IV and at 12 months in the Mayo cohort with similar results (Supplementary Figs. 4 and 5).

We compared the CHOR model (group 1 vs. 2) at the 6month time-point to (1) the HR criteria (achieving CR vs. not) and (2) achieving AOR vs. not using Cox regression, with Mayo cohort as the training cohort and Pavia cohort as the validation cohort. The CHOR model had significantly higher predictive power $(C=0.59)$ compared to the HR model $(C=0.56$; with absolute difference in Harrell's $C$ of $0.03,95 \%$ CI, $0.01-0.06 ; p=0.006$ ) as well as when compared to the OR model $(C=0.56$, with 

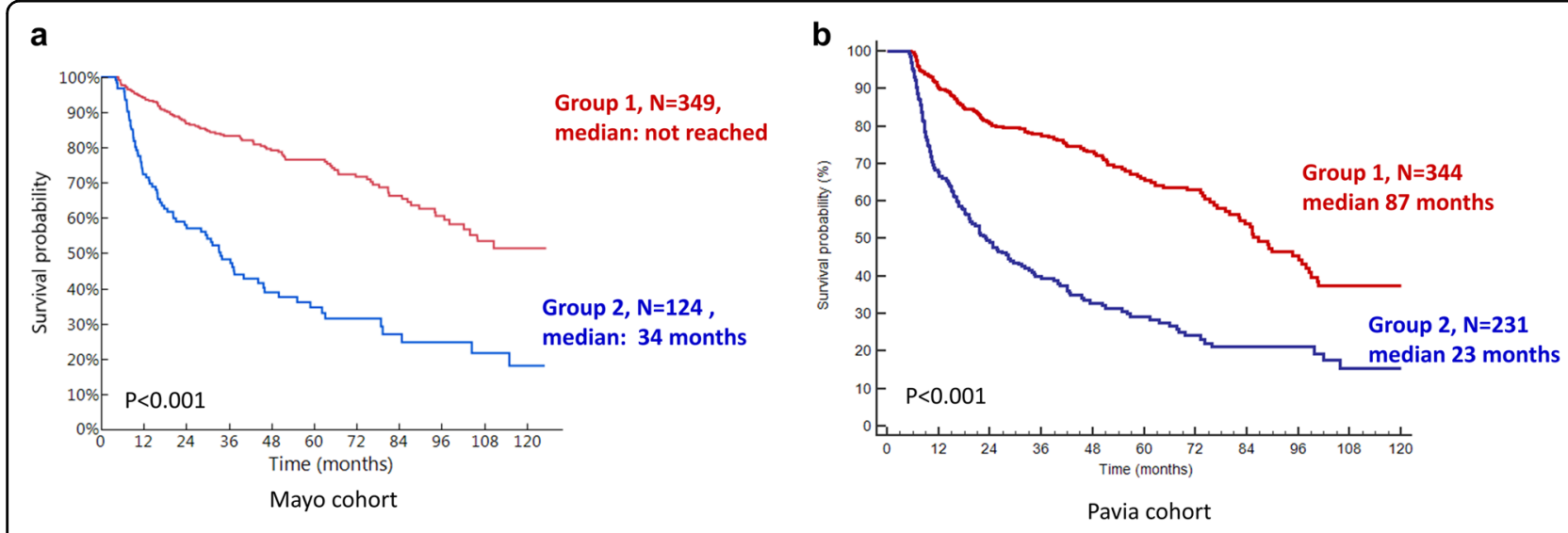

Fig. 4 Overall survival by composite organ and hematologic response (CHOR) model. a Mayo Clinic cohort and b Pavia cohort.

absolute difference in Harrell's $C$ of 0.03 , 95\% CI: $0.01-0.05 ; p=0.009)$. In the subset of patients with cardiac involvement, the CHOR model $(C=0.62)$ had significantly higher predictive value compared with the $\mathrm{HR}$ model $(C=0.57$, with absolute difference in Harrell's $C$ of 0.05 , 95\% CI: $0.02-0.07 ; p=0.001)$; but not when compared to achieving a cardiac response $(C=0.60$, with absolute difference in Harrell's $C$ of $0.02,95 \%$ CI: -0.01 to $0.04 ; p=0.2$ ). In patients with renal involvement, the CHOR model $(C=0.58)$ had significantly higher predictive value when compared with achieving a renal response $(C=0.52$, with absolute difference in Harrell's $C$ of $0.06,95 \%$ CI: $0.02-0.09$; $p=0.2$ ); however this difference was not significant when compared to the HR model $(C=0.56$, with absolute difference of $0.02,95 \% \mathrm{CI}-0.01$ to $0.05 ; p=0.25$ )

\section{Discussion}

At present, there is no available model in AL amyloidosis that allows for concurrent analysis of hematologic and organ responses (especially responses in multiple organs) in a group of patients. Our retrospective study assessed response with treatment in two independent cohorts of patients with $\mathrm{AL}$ amyloidosis to develop and validate a model integrating simultaneous assessment of both HR and OR. Importantly, the model was able to predict OS in both cohorts, with greater predictive power compared with HR or OR assessed in isolation. This model can be used as a surrogate endpoint for rapid assessment of clinical trials. This would allow for shorter duration of follow-up and enable faster completion of these studies. This model can be incorporated in studies designed to make early treatment changes based on response. It can also be easily integrated into clinical practice for prognostication and integrating data across different therapeutics for clinical decision making.
The testing and validation cohorts were large independent cohorts from amyloidosis referral centers with longterm follow-up data. The majority of patients in both cohorts had cardiac involvement and about one-half had involvement of more than one major organ. Overall treatment patterns observed in our cohorts are similar to other cohorts reported over this time period ${ }^{27,30}$. Rates of transplant were strikingly different in the two cohorts (Mayo: 41\%, Pavia: 1\%), which suggests that the results and the CHOR model are generalizable to patients managed with different treatment approaches.

As there is no current method to evaluate multiple organ responses simultaneously, we first developed a combined parameter to assess OR. In both cohorts, patients who achieved response in all organs (AOR) had significantly better OS than those achieving response in some (MOR) or none (NOR) of the involved organs. When comparing MOR and NOR subgroups, there was no difference in OS in the overall Mayo Clinic cohort. However, there was clearly a significant difference MOR vs. NOR groups when evaluating patients with more than one organ involvement, which is the group where mixed or discordant organ responses are possible. This OR endpoint was then combined with HR in a simple, easy to use CHOR model which scored patients from low to high if they achieved response vs. not. This scoring was derived from HR for survival from Cox proportional hazards analysis. Patients could be categorized into two distinct groups with different survival outcomes based on OR and HR assessment at the 6-month landmark in both cohorts. This model was able to distinguish between patients at the 12-month landmark as well. Moreover, this composite model had better predictive power for OS than either HR or OR in isolation in both the test and validation cohorts. The absolute survival outcomes in various groups were different in the Mayo Clinic and Pavia cohort. These differences are likely attributable to several factors 
including the differences in treatment, specifically the rates of stem cell transplant, which were strikingly different $(41 \%$ vs. $1 \%)$ and possibly the responses in individual organs, particularly cardiac response, which is the major driver of survival in AL amyloidosis. Further, while the absolute survival outcomes differed in the two cohorts, the general magnitude of difference was similar.

In subset analysis of patients with cardiac involvement, the composite model had better predictive value compared with HR, but not cardiac response. This may be due to lack of adequate power or alternatively, cardiac response may be the main driving factor impacting survival. On the other hand, in the subset of patients with renal involvement, the CHOR model performed better compared to achieving renal response in predicting patient survival, but not when compared to HR. This may again be due to lack of adequate power or the fact that achieving renal response does not impact $\mathrm{OS}^{27}$. However, renal response remains important as it is a strong predictor for renal failure requiring dialysis as shown in our study and prior reports ${ }^{27}$. As over two-thirds of patients with AL amyloidosis can have involvement of more than one organ ${ }^{4}$, the combined CHOR model, with superior predictive value would be applicable to all patients with AL amyloidosis.

Overall, patients who achieve both OR and HR early in disease course have superior outcomes. This finding is reassuring and the development of a model which can be used systematically to assess both responses is a novel contribution of our study. The current model is able to integrate the relative improvements in hematological and organ parameters to provide a unified readout that can be used in clinical trials, as well as in clinical practice for potentially altering treatment approaches. Our study has limitations given its retrospective design, and heterogeneous nature of treatment received by patients. Moreover, the survival outcomes of patients in the two cohorts are different, likely related to baseline risk and differences in therapies used. However, development of a model in a real world scenario with an independent validation cohort results in wider applicability of the model. Previous cohorts of patients that have been used for development of $\mathrm{HR}$ and OR criteria for amyloidosis have also been treated in a heterogeneous manner ${ }^{16,27}$.

In conclusion, we have developed a model in AL amyloidosis to assess multiple organs concurrently, as well as integrate both HR and OR assessments to determine early clinical benefit with treatment, supporting its use as a surrogate end-point in clinical trials and compare outcomes with different therapeutic approaches. Future studies incorporating this endpoint should be designed to evaluate the utility of changing treatment in patients not achieving this endpoint.

\section{Author details \\ ${ }^{1}$ Division of Hematology, Department of Internal Medicine, Mayo Clinic, Rochester, MN, USA. ${ }^{2}$ Department of Medicine, Stanford University, Stanford, CA, USA. ${ }^{3}$ Amyloidosis Research and Treatment Center, Foundation "Istituto di Ricovero e Cura a Carattere Scientifico (IRCCS) Policlinico San Matteo", Pavia, Italy. ${ }^{4}$ Department of Molecular Medicine, University of Pavia, Pavia, Italy}

\section{Author contributions}

S.S.: Designed the study, collected the data, performed the analysis and wrote the manuscript. P.M.: Collected the data and performed the analysis, critically appraised the manuscript. M.B.: Performed the analysis and critically appraised the manuscript. M.B.: Collected the data and critically appraised the manuscript. N.T.: Collected the data and critically appraised the manuscript. A.F.: Collected the data and critically appraised the manuscript. A.D.: Critically appraised the manuscript. M.A.G.: Critically appraised the manuscript. S.R.H.:

Critically appraised the manuscript. F.K.B.: Critically appraised the manuscript. M.Q.L.: Critically appraised the manuscript. P.K.: Critically appraised the manuscript. N.L.: Critically appraised the manuscript. S.V.R.: Critically appraised the manuscript. G.M.: Critically appraised the manuscript. G.P.: Collected the data, performed the analysis, and critically appraised the manuscript. S.K.K.: Designed the study, performed the analysis and wrote the manuscript.

\section{Conflict of interest}

S.S.: Honoraria/consultancy: Janssen; P.M.: Honoraria/consultancy: Janssen and Pfizer. P.M. is partly supported by a fellowship grant from Collegio Ghislieri (Pavia); A.D.: Consultant for Takeda; Research funding from Celgene, Takeda, Janssen; GlaxoSmithKline, Alnylam, and Pfizer; M.A.G.: Honoraria/consultancy from Ionis, Alnylam, Prothena, Celgene, Janssen, Specytrum, Annexon, Apellis, Amgen, Medscape, Abbvie, Research to Practice, Physcians Education Resource and Teva; P.K.: Research funding (Takeda, Celgene and Amgen); N.L.: Consultancy (Prothena); G.P.: Honoraria/consultancy: Celgene, Janssen, Prothena, Sebia; S.K.K.: Research Funding and membership on an entity's Board of Directors or advisory committees: AbbVie, Celgene, Janssen KITE, Merck. Membership on an entity's Board of Directors or advisory committees:

Oncopeptides, Takeda. Research funding from Novartis and Roche; Remaining authors: none.

\section{Publisher's note}

Springer Nature remains neutral with regard to jurisdictional claims in published maps and institutional affiliations.

Supplementary Information accompanies this paper at (https://doi.org/ 10.1038/s41408-020-0306-5).

Received: 30 August 2019 Revised: 9 March 2020 Accepted: 19 March 2020 Published online: 14 April 2020

\section{References}

1. Gertz, M. A. Immunoglobulin light chain amyloidosis: 2016 update on diagnosis, prognosis, and treatment. Am. J. Hematol. 91, 947-956 (2016).

2. Dispenzieri, A. et al. Treatment of immunoglobulin light chain amyloidosis: Mayo Stratification of Myeloma and Risk-Adapted Therapy (mSMART) consensus statement. Mayo Clinic Proc. 90, 1054-1081 (2015).

3. Merlini, G. et al. Systemic immunoglobulin light chain amyloidosis. Nat. Rev. Dis. Prim. 4, 38 (2018).

4. Muchtar, E. et al. A modern primer on light chain amyloidosis in 592 patients with mass spectrometry-verified typing. Mayo Clinic Proc. 94, 472-483 (2019).

5. Kaufman, G. P. et al. Kinetics of organ response and survival following normalization of the serum free light chain ratio in AL amyloidosis. Am. J. Hematol. 90, 181-186 (2015).

6. Kumar, S. et al. Revised prognostic staging system for light chain amyloidosis incorporating cardiac biomarkers and serum free light chain measurements. J. Clin. Oncol. 30, 989-995 (2012).

7. Dispenzieri, A. et al. Serum cardiac troponins and N-terminal pro-brain natriuretic peptide: a staging system for primary systemic amyloidosis. J. Clin. Oncol. 22, 3751-3757 (2004). 
8. Kourelis, T. V. et al. Coexistent multiple myeloma or increased bone marrow plasma cells define equally high-risk populations in patients with immunoglobulin light chain amyloidosis. J. Clin. Oncol. 31, 4319-4324 (2013).

9. Gertz, M. A. et al. Stem cell transplantation compared with melphalan plus dexamethasone in the treatment of immunoglobulin light-chain amyloidosis. Cancer 122, 2197-2205 (2016).

10. Jaccard, A. et al. High-dose melphalan versus melphalan plus dexamethasone for AL amyloidosis. New Engl. J. Med. 357, 1083-1093 (2007).

11. Mikhael, J. R. et al. Cyclophosphamide-bortezomib-dexamethasone (CyBorD) produces rapid and complete hematologic response in patients with $\mathrm{AL}$ amyloidosis. Blood 119, 4391-4394 (2012).

12. Palladini, G. et al. A European collaborative study of cyclophosphamide, bortezomib, and dexamethasone in upfront treatment of systemic AL amyloidosis. Blood 126, 612-615 (2015).

13. Palladini, G. et al. Oral melphalan and dexamethasone grants extended survival with minimal toxicity in $\mathrm{AL}$ amyloidosis: long-term results of a riskadapted approach. Haematologica. 99, 743-750 (2014).

14. Palladini, G. et al. The combination of high-sensitivity cardiac troponin T (hs(TnT) at presentation and changes in $\mathrm{N}$-terminal natriuretic peptide type $\mathrm{B}$ (NT-proBNP) after chemotherapy best predicts survival in AL amyloidosis. Blood 116, 3426-3430 (2010).

15. Kumar, S. et al. Serum immunoglobulin free light-chain measurement in primary amyloidosis: prognostic value and correlations with clinical features. Blood 116, 5126-5129 (2010).

16. Palladini, G. et al. New criteria for response to treatment in immunoglobulin light chain amyloidosis based on free light chain measurement and cardiac biomarkers: impact on survival outcomes. J. Clin. Oncol. 30, 4541-4549 (2012).

17. Lebovic, D. et al. Predictors of survival in patients with systemic light-chain amyloidosis and cardiac involvement initially ineligible for stem cell transplantation and treated with oral melphalan and dexamethasone. Br. J. Haematol. 143, 369-373 (2008).

18. Gertz, M. A. et al. First-in-Human Phase $1 / /$ study of NEOD001 in patients with light chain amyloidosis and persistent organ dysfunction. J. Clin. Oncol. 34, 1097-1103 (2016).
19. Gertz, M. A., Landau, H. J. \& Weiss, B. M. Organ response in patients with AL amyloidosis treated with NEOD001, an amyloid-directed monoclonal antibody. Am. J. Hematol. 91, E506-E508 (2016).

20. Fernandez de Larrea, C. et al. A practical approach to the diagnosis of systemic amyloidoses. Blood 125, 2239-2244 (2015).

21. Vrana, J. A. et al. Classification of amyloidosis by laser microdissection and mass spectrometry-based proteomic analysis in clinical biopsy specimens. Blood 114, 4957-4959 (2009).

22. Dittrich, $\mathrm{T}$. et al. AL amyloidosis patients with low amyloidogenic free light chain levels at first diagnosis have an excellent prognosis. Blood 130, 632-642 (2017).

23. Milani, P. et al. Patients with light-chain amyloidosis and low free light-chain burden have distinct clinical features and outcome. Blood 130, 625-631 (2017).

24. Sidana, S. et al. Clinical presentation and outcomes in light chain amyloidosis patients with non-evaluable serum free light chains. Leukemia 32, 729-735 (2018).

25. Gertz, M. A. et al. Definition of organ involvement and treatment response in immunoglobulin light chain amyloidosis (AL): a consensus opinion from the 10th International Symposium on Amyloid and Amyloidosis, Tours, France, 18-22 April 2004. Am. J. Hematol. 79, 319-328 (2005).

26. Comenzo, R. L. et al. Consensus guidelines for the conduct and reporting of clinical trials in systemic light-chain amyloidosis. Leukemia 26, 2317-2325 (2012).

27. Palladini, G. et al. A staging system for renal outcome and early markers of renal response to chemotherapy in $\mathrm{AL}$ amyloidosis. Blood 124, 2325-2332 (2014).

28. Cox DR. Regression models and life-tables. J. R. Stat. Soc. Ser. B 34, 187-220 (1972).

29. Newson, R. B. Comparing the predictive powers of survival models using Harrell's C or Somers' D. Stata J. 10, 339-358 (2010).

30. Lilleness, B., Ruberg, F. L., Mussinelli, R., Doros, G. \& Sanchorawala, V. Development and validation of a survival staging system incorporating BNP in patients with light chain amyloidosis. Blood 133, 215-223 (2019). 